\section{Fragment-based reality check}

\section{By Michael J. Haas, Senior Writer}

Researchers at the Burnham Institute for Medical Research have described a drug discovery technique that combines fragment-based synthetic methods and virtual screening to identify inhibitors of proteinprotein interactions faster and more efficiently than either method alone. ${ }^{1}$ In a proof-of-principle study in human breast cancer cells, reported in the Journal of Medicinal Chemistry, the team identified a selective, lowmicromolar inhibitor of X-linked inhibitor of apoptosis.

Team leader Maurizio Pellecchia, professor of infectious diseases at Burnham, told SciBX that an essential feature of the approach is using NMR to confirm the binding behavior of hits. He said this is particularly useful in the initial rounds of screening, in which the potency of the proteinfragment interaction is less important than the geometry of that interaction.

\section{"This is a very nice piece of work that shows how modeling, when guided by experimental constraints, can greatly facilitate early drug discovery."}

- Tom Tibbitts, Infinity Pharmaceuticals Inc.
To overcome this drawback, the Burnham researchers developed a hybrid fragment-based approach that combined the ease of virtual screening with synthesis and NMR verification of hits.

The team sought to identify nonpeptide mimetics of second mitochondrial activator of caspases-also known as diablo homolog (Drosophila) (DIABLO; SMAC) - a ligand of the antiapoptotic protein $\mathrm{X}$-linked inhibitor of apoptosis (XIAP). XIAP is upregulated in some cancers.

The SMAC-XIAP binding interaction was a good test case because the four-residue N-terminus of SMAC that is responsible for XIAP binding has been well characterized.

First, researchers constructed a virtual library of about 1,400 derivatives of alanine-the last of four residues (Ala-Val-Pro-Ile or AVPI) at SMAC's N-terminus. They screened this library with computational docking methods and found 15 hits that appeared to bind XIAP_albeit weakly - in a manner analogous to how SMAC's tail binds XIAP. The group synthesized hits and used NMR to measure whether each hit bound XIAP with the affinity and geometry predicted by virtual docking.

Next, they constructed a 900-member virtual library of derivatives of the compound that NMR showed had the strongest binding affinity for XIAP (about $200 \mu \mathrm{M}$ ). A second round of virtual screening and NMR measurement of binding affinities yielded a molecule with a single-digit micromolar binding affinity for XIAP.

After making one minor structural refinement to improve the drug-like nature of the com-

"Even a fragment with a weak binding interaction that still fits the binding pocket is of interest" and NMR can measure those weak interactions - which may be in the millimolar range-better than other spectroscopic methods, he said.

Traditionally, understanding protein-protein interactions-and designing drug compounds to inhibit them-has depended on the detailed structural information obtained by X-ray crystallography on proteinligand or protein-drug cocrystals. But growing such crystals is difficult and time consuming. Thus, despite great strides made to optimize the process (see Box 1, “Chips and drips"), alternative approaches have been developed over time.

Fragment-based discovery involves screening synthetic libraries of small, drug-like molecular fragments for members that show weak interactions with the target protein. Hits are combined into larger structures by chemical synthesis to produce leads with micromolar binding affinity.

By contrast, conventional methods of virtual screening rely on computational modeling of molecule-target interactions, and only those hits computationally predicted to have the best binding affinities are synthesized and tested.

Although virtual screening is "good for narrowing down the pool of candidates to be tested, by itself it cannot predict which molecules will actually be the best binders," Pellecchia said. The reason, he said, is conformational variations in the real-world molecule or target protein that the software might not take into account.
pound-N-methylation of the original alanine anchor-they confirmed the compound's binding affinity and selectivity for XIAP in human breast cancer cells.

\section{Space exploration}

Pellecchia said his team's fragment-based approach allows investigation of more chemical space than is achieved by screening compound libraries.

"With a library of 5,000 to 10,000 fragments, just pairing any two fragments and linking them together in a number of different ways yields millions of possibilities," he said. In contrast, compound libraries have at most a few hundred thousand members.

Tom Tibbitts, a structural biologist at Infinity Pharmaceuticals Inc. thinks the Burnham team's approach is ready for commercial applications. "This is a very nice piece of work that shows how modeling, when guided by experimental constraints, can greatly facilitate early drug discovery," he said.

Infinity's drug discovery engine uses computational approaches in combination with experimental verification by X-ray, NMR and structure-activity relationships.

Tibbitts noted two key features of the approach published in JMC that contributed to its success. "Manual inspection and filtering of the docking results was important to restrain the solutions, to approximate interactions observed in the [AVPI-XIAP] cocrystal structure and not drift too far 
Physicists at Brandeis University and Harvard University have developed a microfluidics device called the Phase Chip that they say can determine the optimum conditions needed to grow protein crystals for X-ray studies in less time and with less protein than other approaches.

The challenge and art of protein crystallization lie in determining the optimum conditions, such as temperature and concentration of protein and concentration of additives needed to solubilize the protein, which will produce defect-free crystals large enough for X-ray studies.

Because the conditions required to crystallize each protein are different, optimization can be a lengthy trial-anderror process that uses up a lot of protein. In addition, the initial formation of a crystal (nucleation) requires relatively high concentrations of protein, whereas the growth of single, defect-free crystals requires relatively low concentrations.

Enter the Phase Chip, which allows conditions driving nucleation and growth to be separated and explored systematically with as little as $10 \mu \mathrm{g}$ of protein.

The device consists of two stacked layers of polydimethylsiloxane, one thick and one thin. The thick upper layer contains an array of microscopic wellsabout 200 per square centimeter - that are each capable of storing a $1 \mathrm{~nL}$ drop of protein solution. The thin lower layer contains an array of reservoirs, arranged in parallel channels so that there are five distinct reservoirs below every well.

Each well is separated from its underlying reservoirs by $15 \mu \mathrm{m}$ of polydimethylsiloxane through which water-but not protein, salt or other materials-can move in either direction.

The water content of the drops in the wells can be varied by passing salt solutions of different concentrations through the channels of reservoirs below. If the salt content of a drop in a well is lower than that of the reservoir, water will move from the well to the reservoir-increasing the protein concentration in the drop until nucleation begins. If the reservoir flow is changed so that it has a lower salt content than the drop above, water will flow into the well, diluting the drop and facilitating growth of a crystal.

The Brandeis-Harvard research team, led by Seth Fraden, professor of physics at Brandeis, first described the Phase Chip and its potential applications in protein crystallization in a paper published last year in the Journal of the American Chemical Society. ${ }^{2}$ In that paper, Fraden's team used the Phase Chip to grow crystals of xylanase and demonstrated the controlled nucleation, growth and dissolution of crystals by recording microscopic movies.

The problem, Fraden told SciBX, was that "the original design is too complex and too expensive for the market." Since then, the team has been working to redesign the Phase Chip to make it simpler for biologists and biochemists to operate.

"Our plan is to make about 10 copies of the simpler chip for labs that will be beta-testing sites," he said.

Fraden has identified four groups in Massachusetts - three structural biology labs at Brandeis and one at the University of Massachusetts Amherst-to serve as beta-test sites and to provide feedback for further refinements. Betatesting will begin in 2009.

Fraden noted the Phase Chip could be used to grow cocrystals of proteins bound to their ligands. However, his group has not yet scaled up results from Phase Chip experiments to actually make protein crystals for X-ray studies.

Initially Fraden's group had material transfer agreements with Novartis AG and certain research institutions to send him proteins for optimization and crystallization. "But physicists are not the best people to do this," he said. "And we don't want to be the ones to do it. We want to export the technology to other labs that have protein crystallization expertise. I think the right thing to do is get the technology into the labs and hands of the biochemists."

Brandeis and Harvard hold an issued patent on the Phase Chip. $\quad-M J H$ away from this experimental data," he said.

Also, the use of NMR to determine whether the compounds actually bound XIAP's binding pocket was important for confirming weakly binding hits and for validating the overall approach.

Tibbitts added that NMR-based competitive binding studies between SMAC's AVPI tail and the lead compound identified by Pellecchia's team "would have added additional supporting evidence that both ligands bind to the same site."

Pellecchia said his team's approach should be able to identify compounds that inhibit many protein-protein interactions. Indeed, since submitting the JMC paper, the team has identified inhibitors of c-Jun $\mathrm{N}$-terminal kinase (JNK) and undisclosed proteases.

The method reported in the paper is not patented, and Pellecchia said his team would make compounds identified with it freely available for research purposes.

"We can't possibly imagine all of the p ssible applications of the compounds and write patents to cover those uses," he said. "So we would be interested in distributing the compounds to academics and companies-or collaborating with them-to characterize the potential of the molecules."

Haas, M.J. SciBX 1(42); doi:10.1038/scibx.2008.1015

Published online Nov. 20, 2008

REFERENCES

1. Huang, J.-W. et al. J. Med. Chem.; published online Oct. 29, 2008; doi:10.1021/jm8006992

Contact: Maurizio Pellecchia, Burnham Institute for Medical Research, La Jolla, Calif.

e-mail: mpellecchia@burnham.org

2. Shim, J. et al. J. Amer. Chem. Soc. published online June 20, 2007; doi:10.1021/ja071820f

Contact: Seth Fraden, Brandeis University, Waltham, Mass.

e-mail: fraden@brandeis.edu

COMPANIES AND INSTITUTIONS MENTIONED

Brandeis University, Waltham, Mass.

Burnham Institute for Medical Research, La Jolla, Calif.

Harvard University, Boston, Mass.

Infinity Pharmaceuticals Inc. (NASDAQ:INFI), Cambridge, Mass.

Novartis AG (NYSE:NVS; SWX:NOVN), Basel, Switzerland

University of Massachusetts Amherst, Amherst, Mass. 